Research Article

\title{
Prevalence and Genetic Characteristics of Human Bocaviruses Detected in Patients with Acute Respiratory Infections in Bulgaria
}

\author{
Neli Korsun (D), ${ }^{1}$ Svetla Angelova $\left(\mathbb{D},{ }^{1}\right.$ Ivelina Trifonova $\left(\mathbb{D},{ }^{1}\right.$ Silvia Voleva ${ }^{(D)}{ }^{1}$ \\ Iliana Grigorova $\mathbb{D},{ }^{1}$ Iren Tzotcheva $\mathbb{D}^{2},{ }^{2}$ Sirma Mileva $\mathbb{D D}^{2}$ and Penka Perenovska $\mathbb{D}^{2}$ \\ ${ }^{1}$ National Laboratory "Influenza and ARI", National Center of Infectious and Parasitic Diseases, 44A Stoletov Blvd, \\ Sofia, Bulgaria \\ ${ }^{2}$ Pediatric Clinic, University Hospital Alexandrovska, Medical University, 1 Georgi Sofiiski Str, Sofia, Bulgaria \\ Correspondence should be addressed to Neli Korsun; neli_korsun@abv.bg
}

Received 6 September 2021; Accepted 28 October 2021; Published 15 November 2021

Academic Editor: Ahmed Majeed Al-Shammari

Copyright (C) 2021 Neli Korsun et al. This is an open access article distributed under the Creative Commons Attribution License, which permits unrestricted use, distribution, and reproduction in any medium, provided the original work is properly cited.

Human bocaviruses (hBoVs) are often associated with acute respiratory infections (ARIs). Information on the distribution and molecular epidemiology of hBoVs in Bulgaria is currently limited. The objectives of this study were to investigate the prevalence and genetic characteristics of hBoVs detected in patients with ARIs in Bulgaria. From October 2016 to September 2019, nasopharyngeal/oropharyngeal swabs were prospectively collected from 1842 patients of all ages and tested for 12 common respiratory viruses using a real-time RT-PCR. Phylogenetic and amino acid analyses of the hBoV VP1/VP2 gene/protein were performed. $\mathrm{HBoV}$ was identified in $98(5.3 \%)$ patients and was the $6^{\text {th }}$ most prevalent virus after respiratory-syncytial virus (20.4\%), influenza $\mathrm{A}(\mathrm{H} 1 \mathrm{~N} 1)$ pdm09 (11.1\%), $\mathrm{A}(\mathrm{H} 3 \mathrm{~N} 2)$ (10.5\%), rhinoviruses (9.9\%), and adenoviruses (6.8\%). Coinfections with other respiratory viruses were detected in $51 \%$ of the $\mathrm{hBoV}$-positive patients. Significant differences in the prevalence of hBoVs were found during the different study periods and in patients of different age groups. The detection rate of $\mathrm{hBoV}$ was the highest in patients aged $0-4$ years (6.9\%). In this age group, $\mathrm{hBoV}$ was the only identified virus in $9.7 \%, 5.8 \%$, and $1.1 \%$ of the children diagnosed with laryngotracheitis, bronchiolitis, and pneumonia, respectively. Among patients aged $\geq 5$ years, hBoV was detected as a single agent in $2.2 \%$ of cases of pneumonia. Phylogenetic analysis showed that all Bulgarian hBoV strains belonged to the $\mathrm{hBoV} 1$ genotype. A few amino acid substitutions were identified compared to the St1 prototype strain. This first study amongst an all-age population in Bulgaria showed a significant rate of $\mathrm{hBoV}$ detection in some serious respiratory illnesses in early childhood, year-to-year changes in the $\mathrm{hBoV}$ prevalence, and low genetic variability in the circulating strains.

\section{Introduction}

Acute respiratory infections (ARIs) are associated with a huge number of disease cases, outpatient visits, and hospitalizations and significant medical and social costs, thus representing a significant burden on healthcare systems and society as a whole. A wide range of viruses from different families cause respiratory tract diseases of varying severity. Human bocaviruses (hBoVs) are relatively recently identified respiratory pathogens belonging to the family Parvoviridae, subfamily Parvovirinae, and genus Bocaparvovirus $[1,2]$. They are small $(20 \mathrm{~nm})$ viruses with a nonenveloped icosahedral capsid and a nonsegmented, single-stranded, negative-sense or positive-sense DNA genome with a length of approximately $\sim 5.3 \mathrm{~kb}$ organized into three open reading frames (ORFs), which encode the nonstructural proteins (NS1-4), the nuclear phosphoprotein NP1, and the structural proteins VP1/VP2, respectively. The two capsid proteins are responsible for the binding to cellular receptors and transporting the genome to the cell nucleus, and they are the main targets for the host immune response. These proteins share a common C-terminal region of 542 amino acids (aa) and differ only in the N-terminal region of VP1 (VP1u) that consists of 129 aa and is the most variable region of the genome [3]. Based on phylogenetic analysis, hBoVs have been divided into 4 genotypes, among which hBoV1 is 
associated most frequently with respiratory tract illnesses, while hBoV2-4 are mainly pathogens of the gastrointestinal tract [4]. HBoV1 occurs in people of all ages, but is most commonly detected in young children with respiratory symptoms. Seroepidemiological studies show that, over $90 \%$ of 4 -year-olds have antibodies to hBoV1 $[5,6]$. Reinfections occur throughout life, which explains the high seroprevalence in older individuals. The detection rate of hBoV-DNA in patients with ARI varies from $1.5 \%$ to $33 \%$ in different countries and studies [3, 7]. HBoVs have been characterized by an unusually high incidence (up to 90\%) of coinfections $[8,9]$. In hBoV-positive samples, other respiratory viruses or bacteria are often detected, which calls into question the true role of $\mathrm{hBoV} 1$ as a respiratory pathogen. Despite the fact that $\mathrm{hBoV} 1$ is detected in asymptomatic individuals and is a frequent participant in coinfections, this virus has been accepted in a large number of studies as a true cause of upper and lower respiratory tract infections, including infections among hospitalized patients [7, 10, 11].

So far, information on the distribution and clinical significance of hBoVs in Bulgaria is very limited. Previous studies examining the prevalence of respiratory viruses in the country have focused on children less than 5 years old, and data are absent for the prevalence of hBoVs among patients over the entire age range. There were no data on the genetic characteristics of these viruses $[12,13]$. The objectives of this study were to investigate the prevalence and circulation pattern of hBoVs among patients of all ages presenting with ARI symptoms and to perform genetic/ amino acid sequence analyses of the VP1/VP2 gene/protein of hBoV strains.

\section{Materials and Methods}

2.1. Patients and Specimen Collection. Patients in the age range of 0-91 years treated for ARI in primary-care facilities or hospitals located in all 28 regions of the country were enrolled in the National Influenza Surveillance Program. ARIs were defined according to the ECDC (https://ecdc. europa.eu/en/infectious-diseases-public-health/surveillanceand-disease-data/eu-case-definitions). Nasopharyngeal and oropharyngeal specimens, both placed into a common sterile viral transport media tube, were prospectively collected from the enrolled patients either during a visit to the doctor or within the first $24 \mathrm{~h}$ of admission. The specimens were taken 1-7 days after the onset of the illness. After collection, swabs were stored at $2^{\circ} \mathrm{C}-8^{\circ} \mathrm{C}$ for up to $72 \mathrm{~h}$ and transported in ice packs to the National Laboratory "Influenza and ARD" which is recognized as a World Health Organization National Influenza Center. Specimens were processed immediately for virus detection or stored at $-80^{\circ} \mathrm{C}$ prior to analysis.

2.2. Molecular Detection of Respiratory Viruses. Viral DNA and RNA were extracted using an ExiPrep Dx Viral DNA/ RNA kit and ExiPrep16DX equipment (BioNeer, Daejeon, Republic of Korea) in accordance with the manufacturer's instructions. Respiratory samples were tested for influenza viruses using a real-time RT-PCR method and the
SuperScript III Platinum One-Step qRT-PCR System (Invitrogen, ThermoFisher Scientific, Waltham, MA, USA). Primers and probes were provided by the International Reagent Resource (IRR), USA. Amplification was performed using a CFX96 thermal cycler (Bio-Rad Laboratories, Inc., Hercules, CA, USA) according to the protocol recommended by CDC-Atlanta, USA [14]. Clinical samples were screened for noninfluenza viruses including respiratorysyncytial virus (RSV), human metapneumovirus (hMPV), parainfluenza viruses (PIV) 1/2/3, rhinoviruses (RV), adenoviruses $(\mathrm{AdV})$, and bocaviruses (BoVs) using singleplex real-time PCR assays and an AgPath-ID One-Step RT-PCR kit (Applied Biosystems, ThermoFisher Scientific, Waltham, MA, USA). The primers, probes, and PCR conditions used in the study were identical to those described previously $[15,16]$. Positive and negative controls were included in each run. The RNAase-P gene served as an internal positive control for human nucleic acid. Clinical samples were tested in separate real-time RT-PCR assays for the RNAase-P gene, which provided verification of RNA integrity and the absence of PCR inhibition. For influenza type A and type B viruses, positive controls were provided by IRR, USA; for other targets, AmpliRun DNA/RNA Amplification Controls (Vircell, Granada, Spain) were used. The sequences of primers and probes, as well as thermocycling conditions, are shown in the supplementary table.

2.3. VP1/VP2 Gene Sequencing. Conventional PCR was performed to amplify a fragment of the hBoV VP1/VP2 gene region. Nucleic acid amplification was carried out using an Eppendorf Mastercycler instrument (Eppendorf, Stevenage, UK) and a Qiagen One-Step RT-PCR kit (Qiagen, Hilden, Germany) with primers/protocol described previously (Supplementary Table (available here)) [17]. The amplified products with a length of 576 base pairs (bp) corresponding to the nucleotide positions 3233-3808 in the genome of strain PK-5510 (accession number FJ170278) were analyzed by electrophoresis on $2 \%$ agarose gels stained with ethidium bromide. The amplicons were extracted and purified with a PureLink Quick Gel Extraction kit (Invitrogen, Thermo Fisher Scientific, Waltham, MA, USA) according to the manufacturer's instructions. The purified amplicons were sequenced in both directions using the BigDye ${ }^{\mathrm{TM}}$ Terminator v1.1 Cycle Sequencing Kit (Applied Biosystems, Thermo Fisher Scientific, Waltham, MA, USA) on an ABI 3730XL (Thermo Fisher Scientific) DNA Analyser.

Partial VP1/VP2 gene nucleotide sequences of hBoV strains analyzed in this study were deposited in GenBank under the accession numbers MW759050-MW759067.

2.4. Phylogenetic Analysis. The VP1/VP2 gene nucleotide sequences of representative strains of all known $\mathrm{hBoV}$ genotypes as well as sequences of human and animal parvoviruses were downloaded from GenBank using the Basic Local Alignment Search Tool (BLAST) (https://blast.ncbi.nlm.nih. gov/Blast.cgi). The sequences obtained in the present study were aligned with the published sequences using the MUSCLE program embedded in Molecular Evolutionary Genetics 
Analysis software (MEGA, version 6.06; https://www. megasoftware.net/). The best fit nucleotide substitution model, Tamura-3 parameter $(\mathrm{T}-92+\mathrm{G})$, was determined using MEGA 6.06. A phylogenetic tree based on the VP1/VP2 gene was constructed using the maximum likelihood method within MEGA 6.06 software. The reliability of the tree topology was evaluated by bootstrapping with 1000 replications. The study strains were genotyped based on clustering with sequences representing known genotypes.

2.5. Deduced Amino Acid Sequence Analysis. Deduced partial amino acid sequences of Bulgarian $\mathrm{hBoV}$ strains were translated with the standard genetic code using MEGA software. To identify amino acid substitutions, the VP1/VP2 protein sequences of the study strains were aligned with the prototype St1 strain.

Putative N-glycosylation sites were predicted using the NetNGlyc 1.0 web server (https://www.cbs.dtu.dk/services/ NetNGlyc) to identify the sequence motif N-X-S/T (sequon), where $X$ can be any amino acid except proline. Only the sites with scores higher than 0.5 were accepted as glycosylated.

2.6. Statistical Analysis. Statistical analyses were performed using GraphPad Prism v. 8.4.1 (GraphPad Software, San Diego, CA, USA). Chi-square and Fisher's exact tests were used for analyzing the following categorical variables: patients' age, sex, clinical features of illness, and incidence of each virus. A $p$ value of $<0.05$ was considered to be statistically significant.

\section{Results}

3.1. Patient Characteristics. This study was conducted from October 2016 to September 2019 and included three influenza seasons, each starting with week 40 of one year and ending with week 20 of the following year. A total of 1842 patients exhibiting symptoms of ARI were enrolled in the study: 498 in the first season, 500 in the second season, 743 in the third season, and 101 between seasons. Twelve percent $(225 / 1842)$ of these patients attended outpatient healthcare centres, and $87.8 \%(1617 / 1842)$ were hospitalized. The patients' ages ranged from 10 days to 91 years (median age 4.2 years). In the target population, $1332(72.3 \%)$ were $0-4$ years old, $170(9.2 \%)$ were 5-17 years old, $75(4.1 \%)$ were $18-64$ years old, and 265 (14.4\%) were $\geq 65$ years old. A total of 1007 $(54.7 \%)$ of the patients were males, and 835 (45.3\%) were females.

3.2. Viral Detection. All the 1842 patients were tested for 12 respiratory viruses mentioned, and 1229 (66.7\%) (130 outpatients and 1099 inpatients) of them were positive for at least one of these pathogens. Monoinfections were detected in $1042(56.6 \%)$ patients; $172(9.3 \%)$ patients were coinfected with two viruses; and $15(0.8 \%)$ were coinfected with 3 viruses. HBoV was identified in 98 (5.3\%) of the examined patients and was the $6^{\text {th }}$ most frequent virus among the respiratory viruses tested, following RSV (20.4\%), influenza
A(H1N1)pdm09 (11.1\%), influenza A(H3N2) (10.5\%), RV (9.9\%), and $\operatorname{AdV}(6.7 \%)$ (Figure 1).

The detection frequency of $\mathrm{hBoV}$ in the first, second, and third winter seasons was $2.4 \%$ (12/498), 9.6\% (48/500), and $3.8 \%$ (28/743), respectively, and in the periods between seasons, it was an average of $8.9 \%$ (9/101). The incidence rates of $\mathrm{hBoV}$ infection among the outpatients and inpatients were $4 \%(9 / 225)$ and 5.5\% (89/1617), respectively, with no statistically significant difference $(p=0.24)$ (Table 1$)$.

In total, $50(51 \%)$ of the $\mathrm{hBoV}$-positive patients were coinfected with one or two additional respiratory viruses. The most frequently identified copathogens were RVs $(n=17)$, followed by RSV $(n=11), \operatorname{AdV}(n=5)$, influenza $\mathrm{A}(\mathrm{H} 1 \mathrm{~N} 1) \mathrm{pdm} 09(n=4)$ and $\mathrm{A}(\mathrm{H} 3 \mathrm{~N} 2)(n=3), \mathrm{PIV} 1(n=2)$, PIV3 $(n=2)$, and HMPV $(n=1)$. There were no cases of coinfections with PIV-2 and influenza type B. Codetection of two or three different viruses in the same clinical sample was found in $45(45.9 \%)$ and $5(5.1 \%) \mathrm{hBoV}$-positive patients, respectively. Among the respiratory viruses studied, AdVs, hBoVs, and RVs were characterized by the highest proportion of coinfections: $58.1 \%, 51 \%$, and $41.2 \%$, respectively (Figure 2).

A higher frequency of $\mathrm{hBoV}$ infections was found between October and March (84.7\% of all detections). HBoV was detected in few or no specimens in the summer months, but the number of samples tested during this period was relatively small. The highest number of hBoVs was identified in specimens obtained in November 2017 (22/81, 27.2\%) and December 2018 (10/60, 16.7\%) (Figure 3). The periods of increased activity of $\mathrm{hBoV}$ overlapped with those of rhinoviruses.

3.3. Distribution of Patients by Age and Sex. Viral respiratory infections were detected in $73.9 \%$ of patients aged $0-4$ years, $57.2 \%$ of patients aged $5-17$ years, $29.3 \%$ of patients aged $18-64$ years, and $46.4 \%$ of patients aged $\geq 65$ years. $\mathrm{HBoV}$ detection varied in patients aged between 3 months and 82 years. The incidence of $\mathrm{hBoV}$ infection was the highest among the youngest age group (0-4 years) $(6.9 \%)$ and the lowest among the $\geq 65$ years age group $(0.4 \%)$. No hBoVs were detected in patients aged 18-64 years (Table 1). Children aged $0-4$ years represented $72.3 \%$ of the patients studied, but accounted for $93.9 \%$ of the hBoV-positive cases $(p<0.05)$. In the age group of 5-17 years, three of the hBoVpositive patients were 5 years old and two were 6 years old. Among the patients $\geq 65$ years of age, the only $\mathrm{hBoV}$-positive patient was an 82-year-old man with serious breathing difficulties (emphysema). Coinfections of hBoV were detected only in children aged $0-4$ years. Of the $98 \mathrm{hBoV}$ positive patients, $60(61.2 \%)$ were males and 38 (38.8\%) were females without a statistically significant difference $(p=0.21)$.

3.4. Clinical Characteristics. The contribution of $\mathrm{hBoV}$ and other tested respiratory viruses to the development of certain clinical syndromes, laryngotracheitis, bronchiolitis, pneumonia, and central nervous system (CNS) involvement (febrile seizures, cerebral oedema, aseptic meningitis, and 

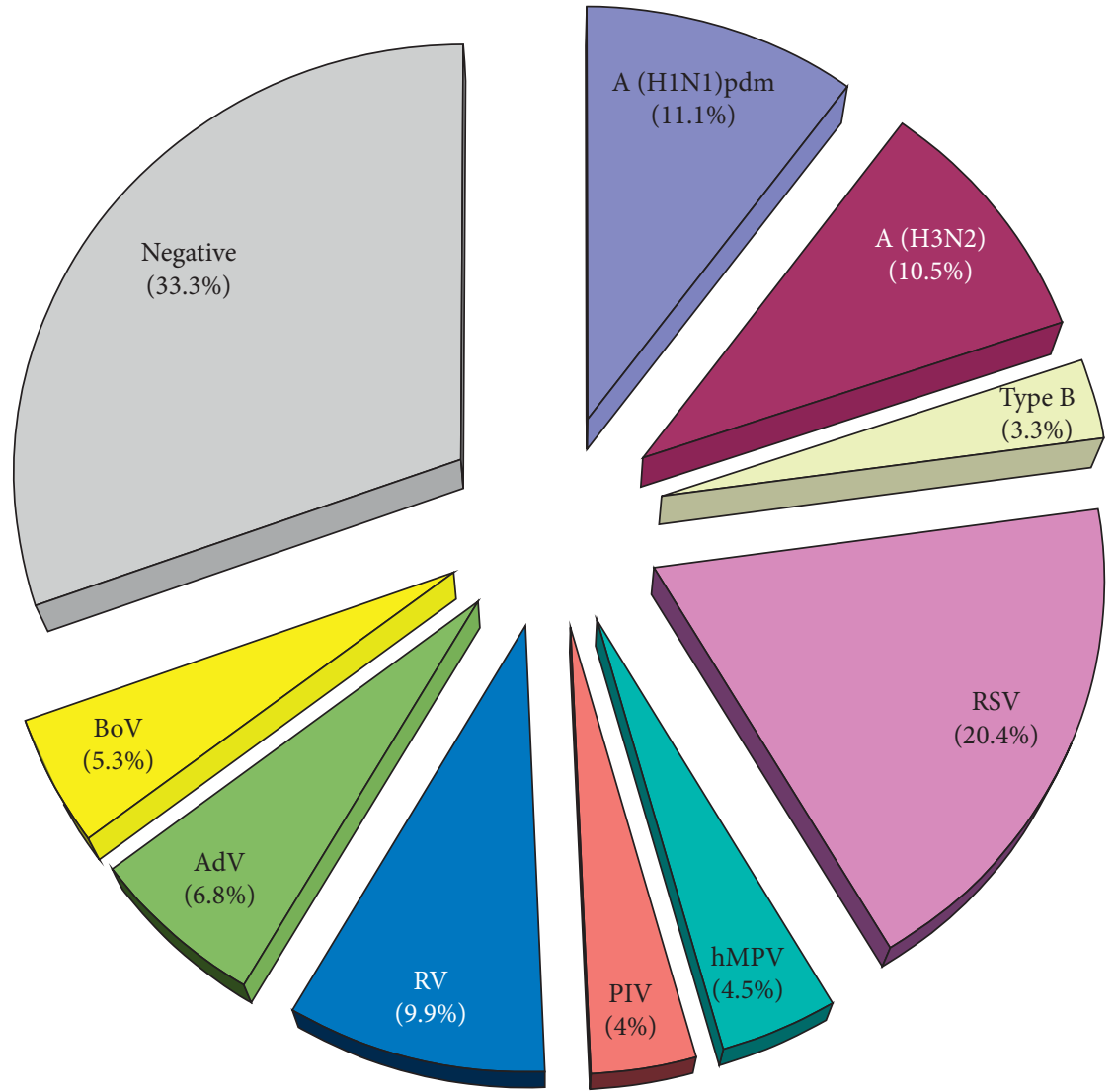

FIgURE 1: Distribution of different respiratory viruses detected in patients with ARI, Bulgaria, 2016-2019.

TABle 1: Distribution of hBoV-positive cases among outpatients and inpatients.

\begin{tabular}{lccc}
\hline Age groups (years) & Outpatients $(\%)(n / N)$ & Inpatients $(\%)(n / N)$ & Total $(\%)(n / N)$ \\
\hline $0-4$ & $4.4(7 / 160)$ & $7.3(85 / 1172)$ & $6.9(92 / 1332)$ \\
$5-17$ & $8.3(2 / 24)$ & $2.1(3 / 146)$ & $2.9(5 / 170)$ \\
$18-64$ & $0(0 / 15)$ & $0(0 / 60)$ & $0(0 / 75)$ \\
$\geq 65$ & $0(0 / 25)$ & $0.4(1 / 240)$ & $0.4(1 / 265)$ \\
Total & $4.0(9 / 225)$ & $5.5(89 / 1617)$ & $5.3(98 / 1842)$ \\
\hline
\end{tabular}

encephalopathy), was analyzed. A total of $113,346,186$, and 43 cases of laryngotracheitis, bronchiolitis, pneumonia, and CNS complications were diagnosed among the children aged 0-4 years, respectively. The hBoV was the second most commonly detected virus in patients with the diagnosis of laryngotracheitis $(19 / 113,16.8 \%)$, third in patients with the diagnosis of bronchiolitis $(38 / 346,11 \%)$, and sixth in patients with the diagnosis of pneumonia (9/146, 4.8\%). The incidence of the $\mathrm{hBoV}$ as a single agent in children diagnosed with laryngotracheitis, bronchiolitis, and pneumonia was $9.7 \%, 5.8 \%$, and $1.1 \%$, respectively. Among the patients aged $\geq 5$ years, hBoV alone was identified in $2.2 \%(2 / 91)$ of the cases of pneumonia (Table 2). No hBoV infections were detected in patients with neurologic complications.

3.5. Phylogenetic Analysis of $h B o V$. For phylogenetic analysis, a fragment of the VP1/VP2 gene from $18 \mathrm{hBoV}$-positive clinical specimens was sequenced. The remaining positive samples had poor or failed PCR amplification or sequencing, probably due to the greater sensitivity of real-time PCR compared to conventional PCR for genotyping or due to low viral load in some samples. The $\mathrm{hBoV}$ sequences were obtained from hospitalized children aged 3 months -6 years with lower respiratory tract infections and from different regions of the country. Phylogenetic analysis showed that all $\mathrm{hBoV}$ strains isolated in this study belonged to the $\mathrm{hBoV} 1$ genotype and hBoV2-4 were not detected. The Bulgarian strains were grouped with the original strain St1 (GenBank access number DQ000495), identified by Allander et al. in 2005 (Figure 4). The hBoV1 sequences identified in this study showed $98.9 \%-100 \%$ homology at the nucleotide level and $97.8 \%-100 \%$ homology at the amino acid level.

3.5.1. Deduced Amino Acid Sequence Analysis. The deduced amino acid sequences of 18 Bulgarian hBoVs were aligned and compared with the sequence of the prototype strain 


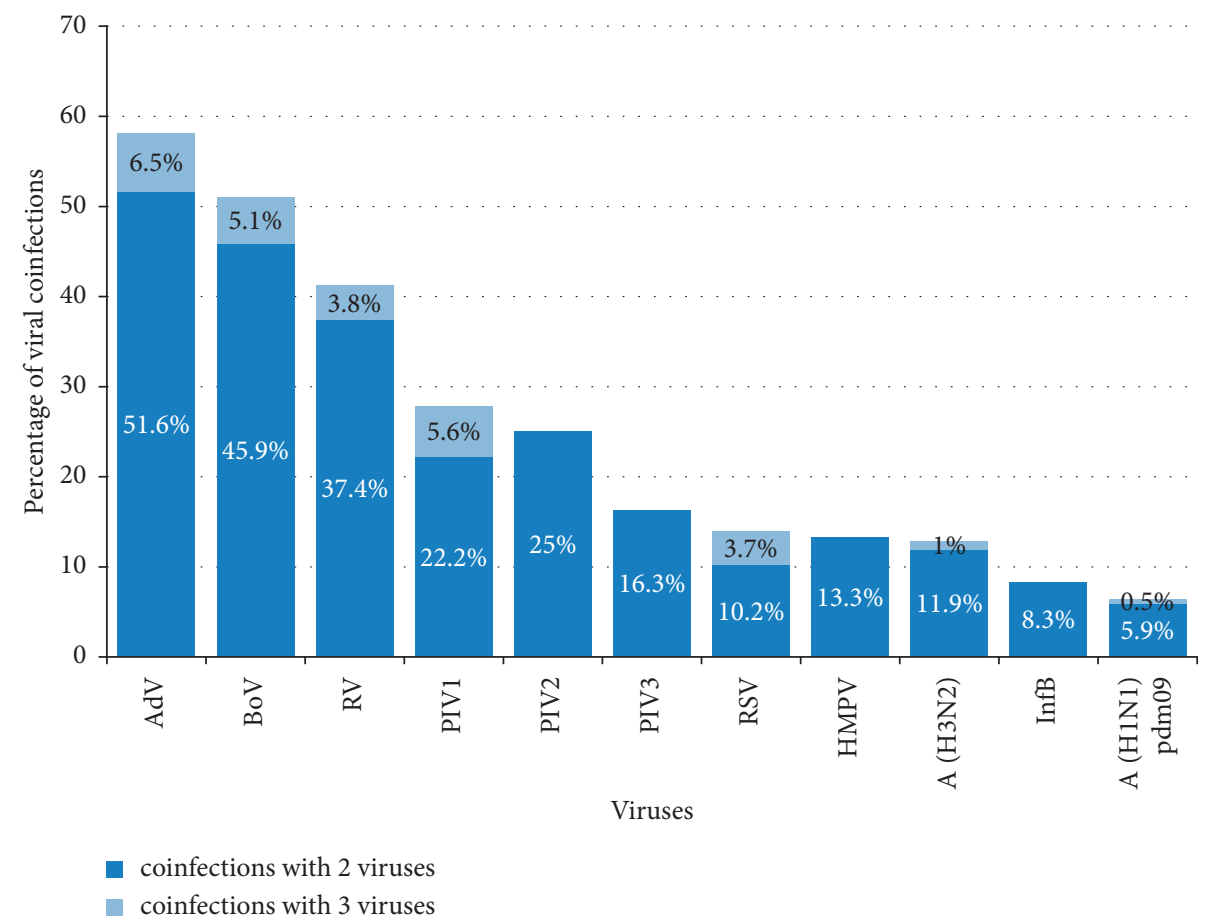

FIgURe 2: Distribution of identified respiratory viruses in cases of coinfections. The percentage of coinfections was calculated based on the total number of infections for each virus.

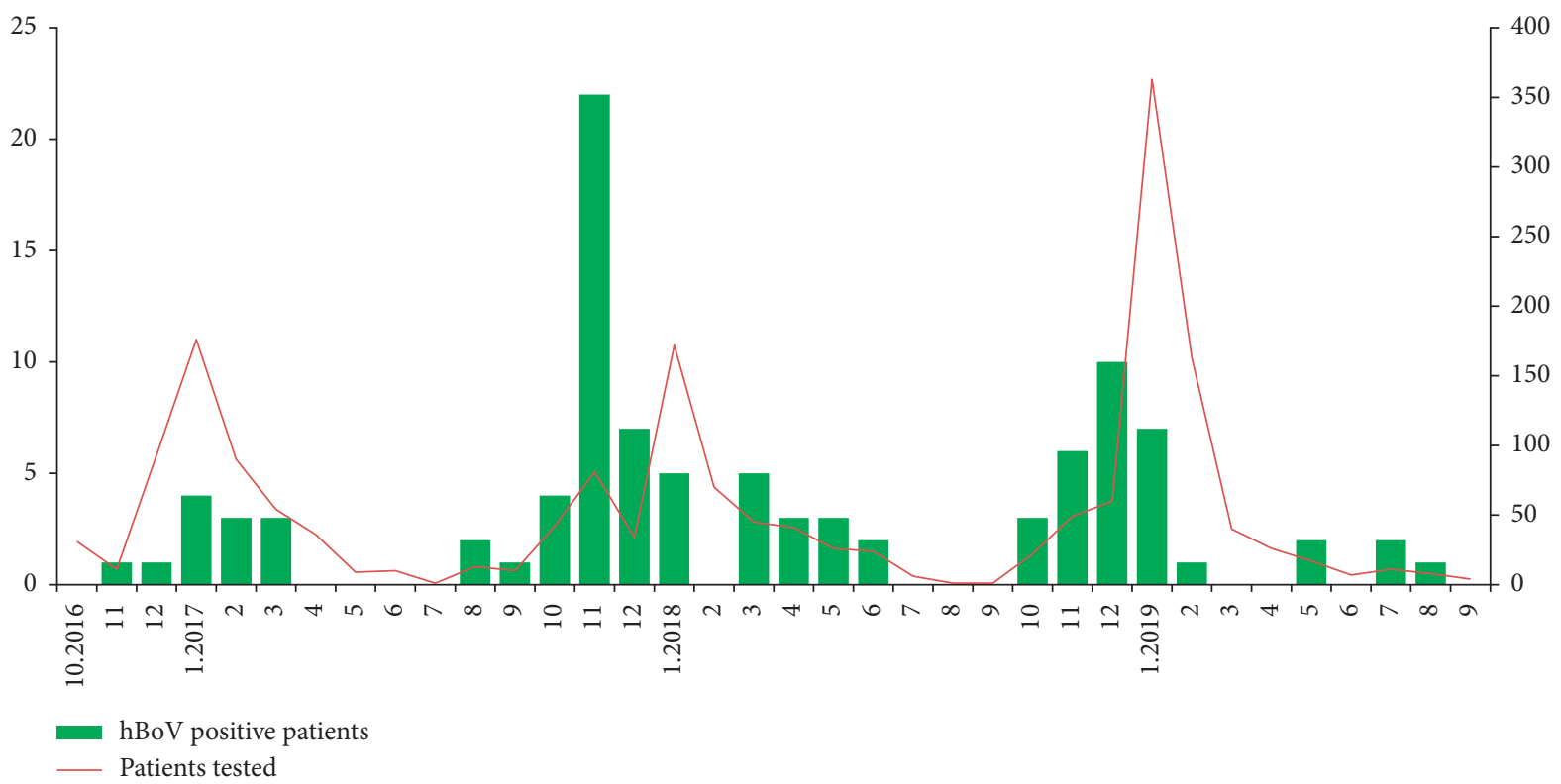

FIgURe 3: Monthly distribution of hBoV detections in Bulgaria, 2016-2019.

St1 (Figure 5). In the VP1/VP2 protein, all Bulgarian hBoV sequences carried two amino acid substitutions: A149T and R251 K. Ten (55.5\%) sequences carried the substitution D180 N, and six (33.3\%) sequences carried the substitutions $\mathrm{I} 264 \mathrm{~K} / \mathrm{R}$. Single amino acid changes were found in $8(44.4 \%)$ sequences in the area of the unique (VP1U) region, corresponding to the first 129 aa at the N-terminus of the VP1 protein. A total of $16.7 \%(3 / 18)$ of the Bulgarian sequences contained 2 amino acid substitutions relative to the prototype hBoV1 strain St1, $27.8 \%$ (5/18) sequences contained 3 substitutions, $38.9 \%$ (7/18) sequences contained 4 substitutions, and 16.7\% (3/ 18) contained 5 substitutions in the studied fragment of the VP1/VP2 protein. All Bulgarian sequences harbored at least two substitutions compared to the St1 strain. The Bulgarian hBoV sequences analyzed possessed one potential N-linked glycosylation site, NTS, at positions $148-150$. 
TABLe 2: Clinical diagnosis among the $\mathrm{hBoV}$-infected patients according to age.

\begin{tabular}{|c|c|c|c|c|c|c|}
\hline \multirow[b]{2}{*}{$\begin{array}{l}\text { Age groups } \\
\text { (years) }\end{array}$} & \multirow[b]{2}{*}{ Total (\%) } & \multicolumn{5}{|c|}{ Clinical diagnosis } \\
\hline & & Laryngotracheitis (\%) & Bronchiolitis (\%) & Pneumonia (\%) & $\begin{array}{c}\text { Other respiratory syndromes } \\
(\%)\end{array}$ & $\begin{array}{c}\text { CNS complications } \\
(\%)\end{array}$ \\
\hline $0-4$ & $92(93.9)$ & $19(20.7)$ & $38(41.3)$ & $9(14.1)$ & $26(28.3)$ & 0 \\
\hline $5-17$ & $5(5.1)$ & 0 & 0 & $2(40)$ & $3(60)$ & 0 \\
\hline $18-64$ & 0 & 0 & 0 & 0 & 0 & 0 \\
\hline$\geq 65$ & $1(1)$ & 0 & 0 & 0 & $1(63)$ & 0 \\
\hline Total & 98 & $19(19.4)$ & $38(38.8)$ & $11(11.2)$ & $30(30.6)$ & 0 \\
\hline
\end{tabular}

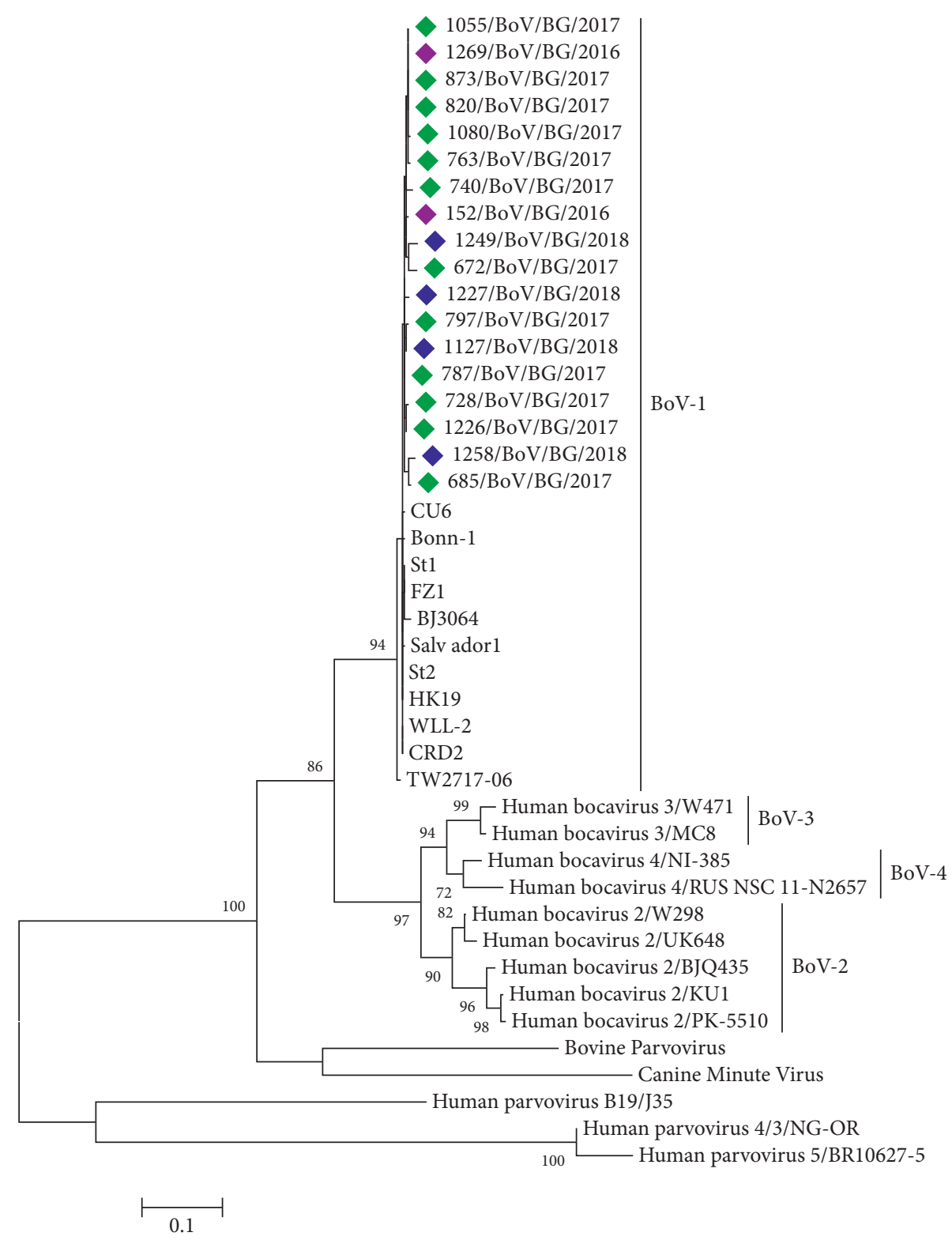

FIGURE 4: Phylogenetic tree based on a fragment of the VP1/VP2 gene of hBoV and other parvoviruses. Genetic distances were measured with the Tamura-3 model with gamma distribution $(\mathrm{T} 92+\mathrm{G})$. The phylogenetic tree was constructed with the maximum likelihood (ML) algorithm using MEGA software (version 6.0). The sequence of prototype hBoV strain St1 is shown in bold. The Bulgarian sequences, detected in 2016, 2017, and 2018, are shown by purple, green, and blue rhombuses, respectively. 

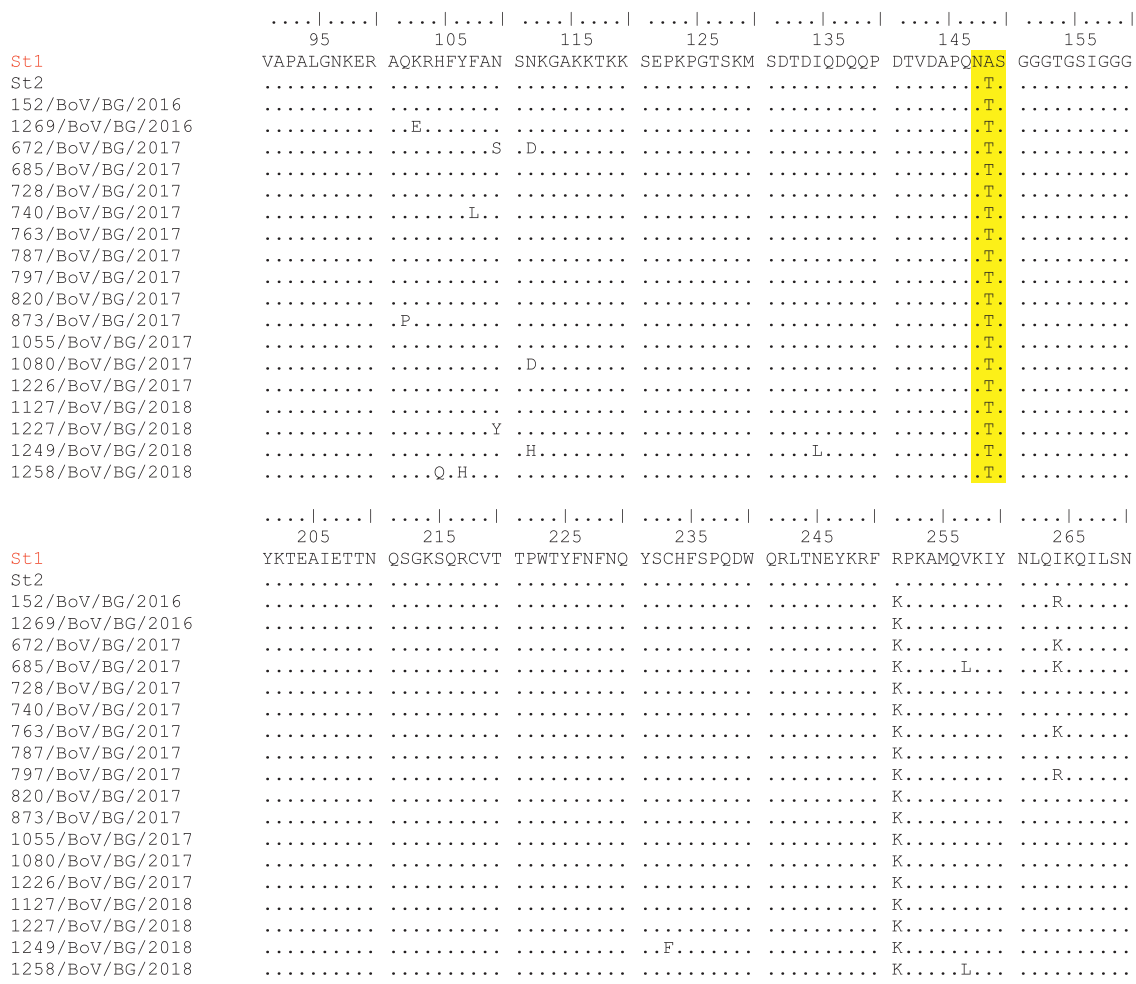

Figure 5: Partial amino acid sequences of the VP1/VP2 protein from Bulgarian hBoV strains. The alignment is shown relative to the sequence of the prototype St1 strain. Identical residues are identified as dots. Yellow shading highlights the predicted N-glycosylation site.

\section{Discussion}

This study presents the prevalence and genetic characteristics of $\mathrm{hBoVs}$ amongst an all-age population in Bulgaria over three consecutive seasons, as well as the epidemiological and clinical features of $\mathrm{hBoV}$ respiratory tract infections. $\mathrm{HBoV}$ showed a moderate detection rate and was the 6th most prevalent virus among the tested 12 respiratory viruses. The general positive rate of $\mathrm{hBoV}$ was $5.3 \%$, with a significant difference between seasons: $2.4 \%$; $9.6 \%$; and 3.8\% $(p<0.05)$. Annual variations in the incidence of $\mathrm{hBoV}$ have also been observed in other countries [18]. The incidence rate of $\mathrm{hBoV}$ varies in different regions of the world depending on the sensitivity of diagnostic tests used, population studied, geographical location, climate, and time period of the study: 9.9\% (Spain) [9], 12.1\% (Italy) [19], 18\% (the Netherlands) [20], 23.1\% (Croatia) [21], 15.5\% (Japan) [22], 6.33\% and 11.64\% (Argentina) [18], and 3.7\% (South Africa) [23]. Previous studies in Bulgaria reported an $\mathrm{hBoV}$ prevalence of $7 \%$ among children younger than five years $[12,13,24]$. In this study, hBoVs were detected in similar proportions among the outpatients and inpatients $(4 \%$ and $5.5 \%)$. A number of researchers have reported a relatively high incidence of $\mathrm{hBoV}$ infections among hospitalized patients $[9,25]$. In the present study, significant differences were found in the prevalence of $\mathrm{hBoV}$ infection in patients of different ages. In close agreement with other reports, the positivity rate of $\mathrm{hBoV}$ was the highest in the youngest age groups (0-4 years), and $93.9 \%$ of all $\mathrm{hBoV}$ cases occurred in this age group [26]. The youngest child with identified $\mathrm{hBoV}$ was 3 months old, which suggests that infants younger than 3 months are probably protected from $\mathrm{hBoV}$ infection by maternal antibodies. No hBoVs were detected in patients aged 18-64 years. Similar to our data, in a large study performed in Kuwait between 2018 and 2020 with 5941 patients suffering ARI, none of the patients aged 10-29 years old had been infected with $\mathrm{hBoV}$, and a small number of $\mathrm{hBoV}$ infections had been detected in the patients aged 30-64 [27]. The low incidence of $\mathrm{hBoV}$ infections in older children and adults, as shown in different studies, is probably due to life-long immunity acquired from primary $\mathrm{hBoV}$ infection during early childhood $[1,8]$. In our study, no influence of the patients' gender on $\mathrm{hBoV}$ infection was observed in contrast to some reports, in which a higher prevalence of HBoV1 was detected in male patients [26]. Consistent with some reports, $\mathrm{hBoV}$ infection displayed a clear seasonality with the highest activity of the virus in the autumn and winter months $[28,29]$, while other authors report year-round circulation of $\mathrm{hBoV}[30,31]$. However, the smaller number of samples tested during the summer months, mainly from hospitalized patients, must be taken into account. The differences in the seasonality of $\mathrm{hBoV}$ infections are probably due to climatic factors. Information on the seasonal activity of respiratory viruses is important for strengthening surveillance and control measures to reduce the risk of nosocomial infections. 
Previous studies have reported that $\mathrm{hBoV}$ has been associated with lower respiratory tract infections such as bronchiolitis, asthma exacerbation, and pneumonia [9, 29]. In this study, hBoV was the third and the sixth most common viral finding in children aged $0-4$ years diagnosed with bronchiolitis and pneumonia, respectively. No association with CNS infections was established, although some authors report detections of $\mathrm{hBoV} 1$ in the cerebrospinal fluid of children with encephalitis [32].

An important feature of $\mathrm{hBoVs}$, detected in Bulgaria, was their frequent involvement in coinfections. Codetection with other respiratory viruses was found in $51 \%$ of the hBoVpositive samples. A higher frequency of mixed infections could be expected if other respiratory viruses (e.g., coronaviruses) were also screened. A high rate of $\mathrm{hBoV}$ coinfections has been also found in studies in other countries: 75\% (Spain) [9]; 78.1\% (Argentina) [18]; 45.2\% (Italy) [33]; and $66.7 \%$ (Vietnam) [34]. This fact can be explained by the prolonged shedding of hBoVs lasting for months after the primary infection [35], as well as by the frequent presence of these viruses in clinically healthy, asymptomatic individuals, which raises the question of whether hBoV are true pathogens of the respiratory tract or just accompanying viruses of other respiratory pathogens (opportunistic copathogens) [2]. In this study, hBoV was detected as a single viral agent in $9.7 \%, 5.8 \%$, and $1.1 \%$ of the children aged $0-4$ years suffering from laryngotracheitis, bronchiolitis, and pneumonia, respectively. Our results are in line with other studies, in which $\mathrm{hBoV}$ infection has been associated with severe respiratory diseases including bronchiolitis, pneumonia, and asthma exacerbation among young children [20,31]. Zhou et al. have reported severe clinical manifestations and high viral loads of hBoV1 in the absence of other viral agents in previously healthy children [36]. There are reports that $\mathrm{hBoVs}$ are capable of causing even life-threatening illnesses $[37,38]$. Furthermore, Brieu et al. have detected $\mathrm{hBoV}$ in hospitalized children ( $<5$ years of age) with respiratory tract disease but in none of the children in the asymptomatic control group [39]. This finding indicates that hBoV is a true respiratory pathogen and not a harmless bystander.

Phylogenetic analysis showed that all the Bulgarian hBoV identified in the current study belonged to genotype 1 (hBoV1). The other three genotypes hBoV2-4 were not found, although some authors reported a low frequency of detection of these genotypes in respiratory samples $[22,40]$. HBoV2-4 have mainly been identified in stool samples of patients with gastroenteritis and have been very rarely associated with respiratory infections. The current study involved only respiratory samples, which could explain the absence of the genotypes $\mathrm{hBoV} 2-4$. To investigate the divergence of the hBoV genome, a fragment of the variable VP1/VP2 gene of hBoV-positive samples was sequenced and the sequences of capsid proteins were compared with those of the prototype strain St1. The VP1/ VP2 genes were highly preserved with minimal sequence variations among the isolates from the different years. No changing trend in the VP1/VP2 protein sequences was observed during the 3-year study period, although these surface proteins are potentially subjected to selective pressure of the immune response of the host. Weak glycosylation of capsid proteins and high degree of homology with the original hBoV strain St1 were found. The Bulgarian sequences contained 2-5 substitutions in the VP1/VP2 proteins compared to the St1 strain. These results are consistent with published data in other countries [19, 41-43]. Researchers from Italy reported that $7.6 \%$ of the 105 study strains had only one amino acid difference, $30.4 \%$ strains had two amino acid differences, and the remaining strains (61.9\%) had at least three amino acid changes in comparison with the reference strain St1 [33]. The hBoV1 strains identified in Greece contain 5 amino acid substitutions in the VP1 protein, and one strain contains three additional substitutions [44]. Eight amino acid substitutions have been found in Cambodian VP1/VP2 sequences [31]. Based on the weak genetic and amino acid polymorphism, some authors suggest that hBoV infection occurs once in a lifetime because it causes the formation of life-long immunity based on the presence of neutralizing antibodies [45].

\section{Conclusions}

This first study amongst an all-age population in Bulgaria showed a significant frequency of hBoV detection in some serious respiratory illnesses in early childhood, year-to-year changes in the hBoV prevalence, and low genetic variability in the circulating strains. Our results could suggest a pathogenic role of this virus in cases of ARI, but additional methods (e.g., detection of hBoV-DNA and hBoV-specific IgM/IgG antibodies in serum samples) should be included to confirm the causality. Viremia, detection of HBoV1 IgM, and seroconversion of IgG antibodies have been considered by some researchers as diagnostic markers for acute hBoV1induced respiratory illness [46]. Prolonged surveillance of circulating viruses is required to fully clarify the clinical significance of $\mathrm{hBoV}$ infection. Continuous genetic analysis of the detected $\mathrm{hBoV}$ would provide information on the genetic variations and molecular evolution of this infectious agent.

\section{Data Availability}

All data generated or analyzed during this study are included within the article (and its supplementary materials).

\section{Conflicts of Interest}

The authors declare no conflicts of interest.

\section{Acknowledgments}

The authors are grateful to all of the nurses and clinicians involved in this study for their help in collecting clinical specimens and patient information. This work was supported by a financial grant from the National Science Foundation of Bulgaria (Project DH 13-15/20.12.2017).

\section{Supplementary Materials}

Supplementary Table. Primers/probes used in this study and thermocycling conditions. Abbreviations: NCR, noncoding region; F, forward primer; $\mathrm{R}$, reverse primer; $\mathrm{P}$, probe; FAM, 
6-carboxyfluorescein; BHQ, black hole quencher; underlining and boldface indicate a locked nucleic acid. (Supplementary Materials)

\section{References}

[1] T. Allander, M. T. Tammi, M. Eriksson, A. Bjerkner, A. Tiveljung-Lindell, and B. Andersson, "From the Cover: cloning of a human parvovirus by molecular screening of respiratory tract samples," Proceedings of the National Academy of Sciences, vol. 102, no. 36, pp. 12891-12896, 2005.

[2] O. Schildgen, A. Müller, T. Allander et al., "Human bocavirus: passenger or pathogen in acute respiratory tract infections?" Clinical Microbiology Reviews, vol. 21, no. 2, pp. 291-304, 2008.

[3] T. Jartti, K. Hedman, L. Jartti, O. Ruuskanen, T. Allander, and M. Söderlund-Venermo, "Human bocavirus-the first 5 years," Reviews in Medical Virology, vol. 22, no. 1, pp. 46-64, 2012.

[4] M. Guido, M. R. Tumolo, T. Verri et al., "Human bocavirus: current knowledge and future challenges," World Journal of Gastroenterology, vol. 22, no. 39, pp. 8684-8697, 2016.

[5] J. S. Kahn, D. Kesebir, S. F. Cotmore et al., "Seroepidemiology of human bocavirus defined using recombinant virus-like particles," The Journal of Infectious Diseases, vol. 198, no. 1, pp. 41-50, 2008.

[6] M. Guido, A. Zizza, S. Bredl et al., "Seroepidemiology of human bocavirus in Apulia, Italy," Clinical Microbiology and Infections, vol. 18, no. 4, pp. E74-E76, 2012.

[7] S. Esposito, S. Bosis, H. G. M. Niesters et al., "Impact of human bocavirus on children and their families," Journal of Clinical Microbiology, vol. 46, no. 4, pp. 1337-1342, 2008.

[8] A. M. Fry, X. Lu, M. Chittaganpitch et al., "Human bocavirus: a novel parvovirus epidemiologically associated with pneumonia requiring hospitalization in Thailand," The Journal of Infectious Diseases, vol. 195, no. 7, pp. 1038-1045, 2007.

[9] C. Calvo, M. L. García-García, F. Pozo, D. Carballo, E. Martínez-Monteserín, and I. Casas, "Infections and coinfections by respiratory human bocavirus during eight seasons in hospitalized children," Journal of Medical Virology, vol. 88, no. 12, pp. 2052-2058, 2016.

[10] T. Allander, T. Jartti, S. Gupta et al., "Human bocavirus and acute wheezing in children," Clinical Infectious Diseases, vol. 44, no. 7, pp. 904-910, 2007.

[11] R. W. Körner, M. Söderlund-Venermo, S. van KoningsbruggenRietschel, R. Kaiser, M. Malecki, and O. Schildgen, "Severe human bocavirus infection, Germany," Emerging Infectious Diseases, vol. 17, no. 12, pp. 2303-2305, 2011.

[12] N. Korsun, S. Angelova, I. Trifonova et al., "Viral pathogens associated with acute lower respiratory tract infections in children younger than 5 years of age in Bulgaria," Brazilian Journal of Microbiology, vol. 50, no. 1, pp. 117-125, 2019.

[13] N. Korsun, S. Angelova, I. Trifonova et al., "Predominance of ON1 and BA9 genotypes of respiratory syncytial virus (RSV) in Bulgaria, 2016-2018," Journal of Medical Virology, vol. 93, no. 6, pp. 3401-3411, 2021.

[14] B. Shu, K.-H. Wu, S. Emery et al., "Design and performance of the CDC real-time reverse transcriptase PCR swine flu panel for detection of 2009 A (H1N1) pandemic influenza virus," Journal of Clinical Microbiology, vol. 49, no. 7, pp. 2614-2619, 2011.

[15] M. Kodani, G. Yang, L. M. Conklin et al., "Application of TaqMan low-density arrays for simultaneous detection of multiple respiratory pathogens," Journal of Clinical Microbiology, vol. 49, no. 6, pp. 2175-2182, 2011.
[16] X. Lu, M. Chittaganpitch, S. J. Olsen et al., "Real-time PCR assays for detection of bocavirus in human specimens," Journal of Clinical Microbiology, vol. 44, no. 9, pp. 3231-3235, 2006.

[17] A. Kapoor, P. Simmonds, E. Slikas et al., "Human bocaviruses are highly diverse, dispersed, recombination prone, and prevalent in enteric infections," The Journal of Infectious Diseases, vol. 201, no. 11, pp. 1633-1643, 2010.

[18] L. M. Ghietto, D. Majul, P. Ferreyra Soaje et al., "Comorbidity and high viral load linked to clinical presentation of respiratory human bocavirus infection," Archives of Virology, vol. 160, no. 1, pp. 117-127, 2015.

[19] A. Zappa, M. Canuti, E. Frati et al., "Co-circulation of genetically distinct human metapneumovirus and human bocavirus strains in young children with respiratory tract infections in Italy," Journal of Medical Virology, vol. 83, no. 1, pp. 156-164, 2011.

[20] F. M. Moesker, J. J. van Kampen, A. A. van der Eijk et al., "Human bocavirus infection as a cause of severe acute respiratory tract infection in children," Clinical microbiology and infection: The Official Publication of the European Society of Clinical Microbiology and Infectious Diseases, vol. 21, pp. e1-8, 2015.

[21] S. Ljubin-Sternak, T. Meštrović, I. Ivković-Jureković et al., "High detection rates of human bocavirus in infants and small children with lower respiratory tract infection from Croatia," Clinical Laboratory, vol. 65, no. 1, 2019.

[22] N. Koseki, S. Teramoto, M. Kaiho et al., "Detection of human bocaviruses 1 to 4 from nasopharyngeal swab samples collected from patients with respiratory tract infections," Journal of Clinical Microbiology, vol. 50, no. 6, pp. 2118-2121, 2012.

[23] K. Subramoney, O. Hellferscee, M. Pretorius et al., "Human bocavirus, coronavirus, and polyomavirus detected among patients hospitalised with severe acute respiratory illness in South Africa, 2012 to 2013," Health Science Reports, vol. 1, no. 8, p. e59, 2018.

[24] N. S. Korsun, S. G. Angelova, I. T. Trifonova et al., "The prevalence and genetic characterization of human metapneumovirus in Bulgaria, 2016-2019," Intervirology, vol. 64, no. 4, pp. 194-202, 2021.

[25] C. do Amaral de Leon, S. L. Amantea, D. A. Pilger, and V. Cantarelli, "Clinical and epidemiologic profile of lower respiratory tract infections associated with human bocavirus," Pediatric Pulmonology, vol. 48, no. 11, pp. 1112-1118, 2013.

[26] W.-K. Liu, Q. Liu, D.-H. Chen et al., "Epidemiology of HBoV1 infection and relationship with meteorological conditions in hospitalized pediatric patients with acute respiratory illness: a 7-year study in a subtropical region," BMC Infectious Diseases, vol. 18, no. 1, p. 329, 2018.

[27] N. M. Madi and A. Al-Adwani, "Human bocavirus (HBoV) in Kuwait: molecular epidemiology and clinical outcome of the virus among patients with respiratory diseases," Journal of Medical Microbiology, vol. 69, no. 7, pp. 1005-1012, 2020.

[28] F. Pozo, M. L. García-García, C. Calvo, I. Cuesta, P. PérezBreña, and I. Casas, "High incidence of human bocavirus infection in children in Spain," Journal of Clinical Virology, vol. 40, no. 3, pp. 224-228, 2007.

[29] S. Falahi, H. Sayyadi, A. Abdoli, A. Kenarkoohi, and S. Mohammadi, "The prevalence of human bocavirus in $<2$ year-old children with acute bronchiolitis," New Microbes and New Infections, vol. 37, Article ID 100736, 2020.

[30] B. Weissbrich, F. Neske, J. Schubert et al., "Frequent detection of bocavirus DNA in German children with respiratory tract infections," BMC Infectious Diseases, vol. 6, no. 1, p. 109, 2006.

[31] A. Arnott, S. Vong, S. Rith et al., "Human bocavirus amongst an all-ages population hospitalised with acute lower 
respiratory infections in Cambodia," Influenza and Other Respiratory Viruses, vol. 7, no. 2, pp. 201-210, 2013.

[32] M. T. Mitui, S. M. Shahnawaz Bin Tabib, T. Matsumoto et al., "Detection of human bocavirus in the cerebrospinal fluid of children with encephalitis," Clinical Infectious Diseases, vol. 54, no. 7, pp. 964-967, 2012.

[33] N. Principi, A. Piralla, A. Zampiero et al., "Bocavirus infection in otherwise healthy children with respiratory disease," PLoS One, vol. 10, no. 8, Article ID e0135640, 2015.

[34] D. N. Tran, T. Q. N. Nguyen, T. A. Nguyen, S. Hayakawa, M. Mizuguchi, and H. Ushijima, "Human bocavirus in children with acute respiratory infections in vietnam," Journal of Medical Virology, vol. 86, no. 6, pp. 988-994, 2014.

[35] K. Blessing, F. Neske, U. Herre, H.-W. Kreth, and B. Weissbrich, "Prolonged detection of human bocavirus DNA in nasopharyngeal aspirates of children with respiratory tract disease," Pediatric Infectious Disease Journal, vol. 28, no. 11, pp. 1018-1019, 2009.

[36] L. Zhou, S. Zheng, Q. Xiao et al., "Single detection of human bocavirus 1 with a high viral load in severe respiratory tract infections in previously healthy children," BMC Infectious Diseases, vol. 14, no. 1, p. 424, 2014.

[37] T. Ursic, A. Steyer, S. Kopriva, G. Kalan, U. Krivec, and M. Petrovec, "Human bocavirus as the cause of a lifethreatening infection," Journal of Clinical Microbiology, vol. 49, no. 3, pp. 1179-1181, 2011.

[38] N. Edner, P. Castillo-Rodas, L. Falk, K. Hedman, M. Soderlund-Venermo, and T. Allander, "Life-threatening respiratory tract disease with human bocavirus-1 infection in a 4-year-old child," Journal of Clinical Microbiology, vol. 50, no. 2, pp. 531-532, 2012.

[39] N. Brieu, G. Guyon, M. Rodière, M. Segondy, and V. Foulongne, "Human bocavirus infection in children with respiratory tract disease," Pediatric Infectious Disease Journal, vol. 27, no. 11, pp. 969-973, 2008.

[40] J.-R. Song, Y. Jin, Z.-P. Xie et al., "Novel human bocavirus in children with acute respiratory tract infection," Emerging Infectious Diseases, vol. 16, no. 2, pp. 324-327, 2010.

[41] Y. Wang, Y. Li, J. Liu et al., "Genetic characterization of human bocavirus among children with severe acute respiratory infection in China," Journal of Infection, vol. 73, no. 2, pp. 155-163, 2016.

[42] S. Kenmoe, M.-A. Vernet, M. Njankouo-Ripa, V. B. Penlap, A. Vabret, and R. Njouom, "Phylogenic analysis of human bocavirus detected in children with acute respiratory infection in Yaounde, Cameroon," BMC Research Notes, vol. 10, no. 1, p. 293, 2017.

[43] B. Moreno, L. Abrego, J.-P. Carrera et al., "Detection of human bocavirus type 1 infection in Panamanian children with respiratory illness," Journal of Medical Virology, vol. 88, no. 3, pp. 389-394, 2016.

[44] V. Pogka, A. Moutousi, A. Kossyvakis et al., "Genetic variability of human metapneumo- and bocaviruses in children with respiratory tract infections," Influenza and Other Respiratory Viruses, vol. 8, no. 1, pp. 107-115, 2014.

[45] S. K. P. Lau, C. C. Y. Yip, T. 1. Que et al., "Clinical and molecular epidemiology of human bocavirus in respiratory and fecal samples from children in Hong Kong," The Journal of Infectious Diseases, vol. 196, no. 7, pp. 986-993, 2007.

[46] V. Eskola, M. Xu, and M. Söderlund-Venermo, "Severe lower respiratory tract infection caused by human bocavirus 1 in an infant," Pediatric Infectious Disease Journal, vol. 36, no. 11, pp. 1107-1108, 2017. 